\title{
Importance of mediastinal screening-based observation during endoscopic ultrasound to examine gastrointestinal pathologies
}

\author{
Naohito Uchida ${ }^{1} \cdot$ Yuko Bando ${ }^{1} \cdot$ Sae Hamaya ${ }^{1} \cdot$ Yukiko Koyama ${ }^{1} \cdot$ Kazuhiro Kozuka $^{1} \cdot$ Rie Kawakita $^{1}$ Yuki Sawada ${ }^{1}$ \\ Akihiko Tatemoto $^{2} \cdot$ Toshiaki Nakatsu $^{1}$
}

Received: 31 July 2018 / Accepted: 4 September 2018 / Published online: 12 September 2018

(c) The Author(s) 2018

\begin{abstract}
In all endoscopic ultrasound (EUS) examinations performed at our hospital, the heart, vasculature, and mediastinal lymph nodes from the esophagus are observed after checking for gastrointestinal pathologies. Since the introduction of EUS using a convex linear-array echoendoscope at our hospital in April 2015, EUS examinations have been performed in 371 cases for examining pancreaticobiliary diseases, submucosal tumors, and other pathologies during the 3-year period, till March 2018. We diagnosed 2 patients with asymptomatic cardiovascular disease while observing the mediastinum during EUS examination to examine identified pancreaticobiliary disease. No subjective symptoms associated with cardiovascular disease were observed and the respective conditions had not been identified previously in either case. One case involved a left atrial myxoma while the other involved a saccular aortic aneurysm in the thoracic aorta. A left atrial tumor resection and aortic replacement surgery were performed in each case. Their postoperative courses have been favorable. As cardiovascular diseases are often life-threatening, as in the present 2 cases, observational screening of the cardiovascular system from the esophagus should also be performed during EUS examinations just as the pharyngeal region is examined during upper gastrointestinal endoscopy.
\end{abstract}

Keywords Endoscopic ultrasound (EUS) $\cdot$ Screening $\cdot$ Mediastinum $\cdot$ Cardiovascular disease $\cdot$ Myxoma

\section{Introduction}

Endoscopic ultrasound (EUS) is useful in the diagnosis of not only pancreaticobiliary and submucosal tumors, but also mediastinal diseases, such as mediastinal tumors and mediastinal lymph node enlargement $[1,2]$. We diagnosed 2 patients with asymptomatic cardiovascular disease while observing the mediastinum during EUS examination to examine identified pancreaticobiliary disease. One case involved a left atrial myxoma while the other involved a saccular aortic aneurysm in the thoracic aorta. There were no subjective symptoms associated with these cardiovascular pathologies in either case, and the pathologies had not been detected previously. These cases suggest the importance of

Naohito Uchida

naohito@med.kagawa-u.ac.jp

1 Departments of Gastroenterology, Saint Martin's Hospital, 1-4-13 Tani-machi, Sakaide City, Kagawa 762-0033, Japan

2 Departments of Surgery, Saint Martin's Hospital, 1-4-13 Tani-machi, Sakaide City, Kagawa 762-0033, Japan observational cardiovascular screening from the esophagus even in cases where EUS is performed to closely examine gastrointestinal disease.

\section{Case reports}

In all EUS examinations performed at our hospital, the heart, vasculature, and mediastinal lymph nodes from the esophagus are observed after checking for gastrointestinal pathologies. Since the introduction of EUS at our hospital in April 2015, EUS examinations have been performed in 371 cases for examining pancreaticobiliary diseases, submucosal tumors, and other pathologies during the 3-year period, till March 2018. The results of these 371 EUS examinations included 1 case of left atrial myxoma and 1 case of saccular aortic aneurysm of the thoracic aorta. No subjective symptoms associated with cardiovascular disease were observed in either case, and the respective conditions had not been identified previously. A convex linear-array echoendoscope (GF-UGT260; Olympus Optical Corp., Tokyo, 
Japan) connected to an ultrasonography platform (EUME2; Olympus Optical Corp., Tokyo, Japan) was used in this study. All EUS examinations were carried out under conscious sedation with i.v. midazolam by an experienced endosonographer (NU) who had 15 years of experience carrying out the procedure.

Case 1 A 75-year-old woman had been followed-up by our hospital since 2009 via abdominal computed tomographic (CT) examination, magnetic resonance cholangiopancreaticography (MRCP), or other examination methods for intraductal papillary mucinous neoplasm (IPMN). Following its introduction, when EUS was performed as follow-up for IPMN in January 2017, observation of the mediastinum revealed a lesion measuring $15 \mathrm{~mm} \times 9 \mathrm{~mm}$ with heterogeneous echoic pattern and partly calcified pattern at the left atrial wall (Fig. 1). A left atrial tumor was suspected based on a contrast CT scan (Fig. 2) and other considerations. A left atrial tumor resection was performed in April 2017. The tumor was a $20 \mathrm{~mm} \times 15 \mathrm{~mm}$ broad-based tumor attached to the atrial septum. Histopathological examination revealed small circular and spindle-shaped tumor cells in the mucous stroma, in addition to hemosiderin deposits and linear calcification (Gamna-Gandy bodies), which are indicative of myxoma (Fig. 3a, b). Based on these findings, the lesion was determined as left atrial myxoma. The patient has exhibited a favorable postoperative course and continues to be followed-up regularly for IPMN.

Case 2 A 67-year-old man underwent EUS in December 2017 to examine localized gallbladder wall thickening identified at another hospital. Observation from the stomach and duodenum revealed localized wall thickening with Rokitansky-Aschoff sinuses at the base of the gallbladder. This

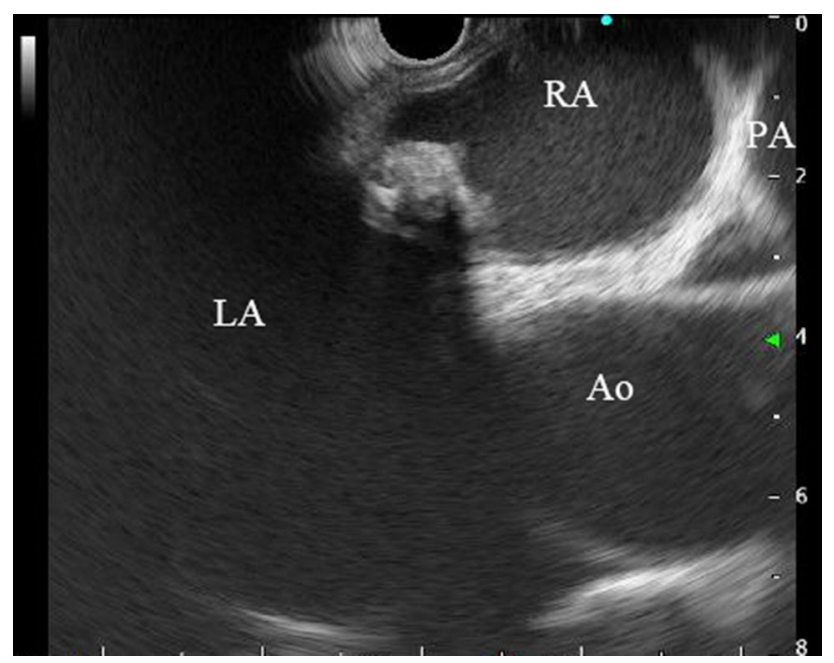

Fig. 1 In endoscopic ultrasound, observation of the mediastinum revealed a lesion measuring $15 \mathrm{~mm} \times 9 \mathrm{~mm}$ with heterogeneous echoic pattern and partly calcified pattern at the left atrial wall. $L A$ left atrium, $R A$ right atrium, $A o$ aorta, $P A$ pulmonary artery

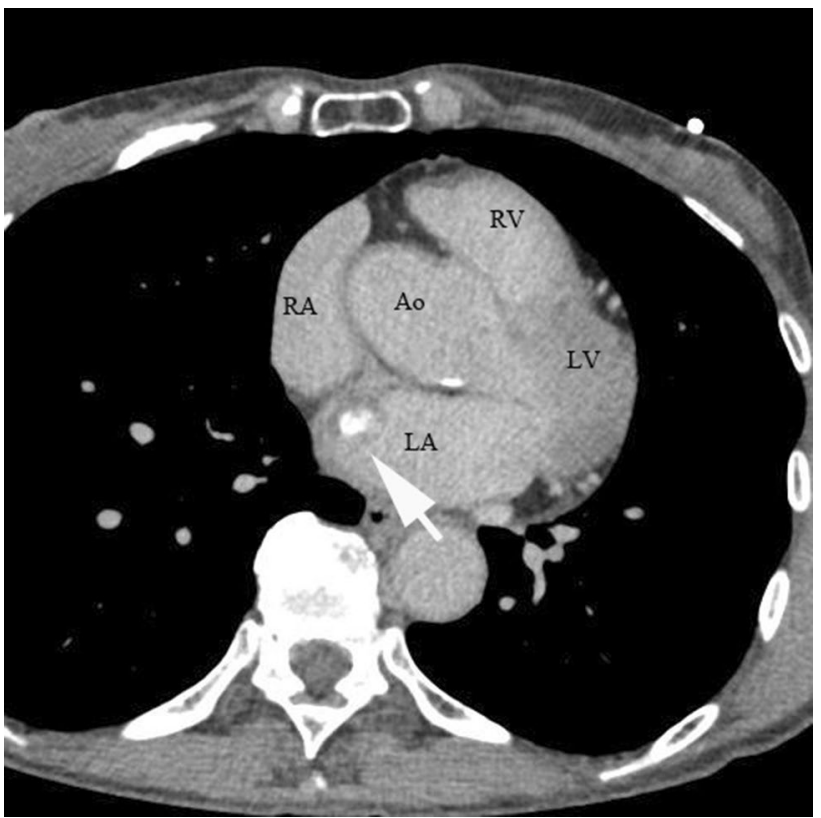

Fig. 2 Enhanced computed tomography demonstrated partly calcified tumor at the left atrial wall (arrow). $R A$ right atrium, $L A$ left atrium, $A o$ aorta, $R V$ right ventricle, $L V$ left ventricle

was diagnosed as localized fundal type adenomyomatosis of the gallbladder. Continued observation of the mediastinum revealed aortic wall thickening and a sac-like protrusion of the aortic lumen, and an aortic aneurysm was suspected (Fig. 4). Contrast CT examination revealed a saccular aneurysm in the aortic arch (Fig. 5), which was determined to be operable. Aortic replacement surgery at the aortic arch was performed in March 2018. The patient's postoperative course has been favorable.

\section{Discussion}

In all EUS examinations performed at our hospital, the mediastinum from the esophagus is observed after checking for gastrointestinal pathologies. The procedure is to first visualize the liver, and subsequently, examine the right atrium from the hepatic vein to the inferior vena cava. Following this, the scope is slightly turned counterclockwise and the left atrium, left ventricle, ascending aorta, pulmonary artery, and the lymph nodes of subcarinal space are observed. Subsequently, the scope is withdrawn and rotated leftward at the site where air was visible through the carina. The aortopulmonary window lymph nodes, aortic arch, and pulmonary artery are then examined. It is important to operate the scope gently during mediastinal observation because the esophagus is limited space compared with the stomach.

Primary cardiac tumor is a rare disease identified in $0.001-0.28 \%$ of all autopsies. Approximately, $70 \%$ of these 

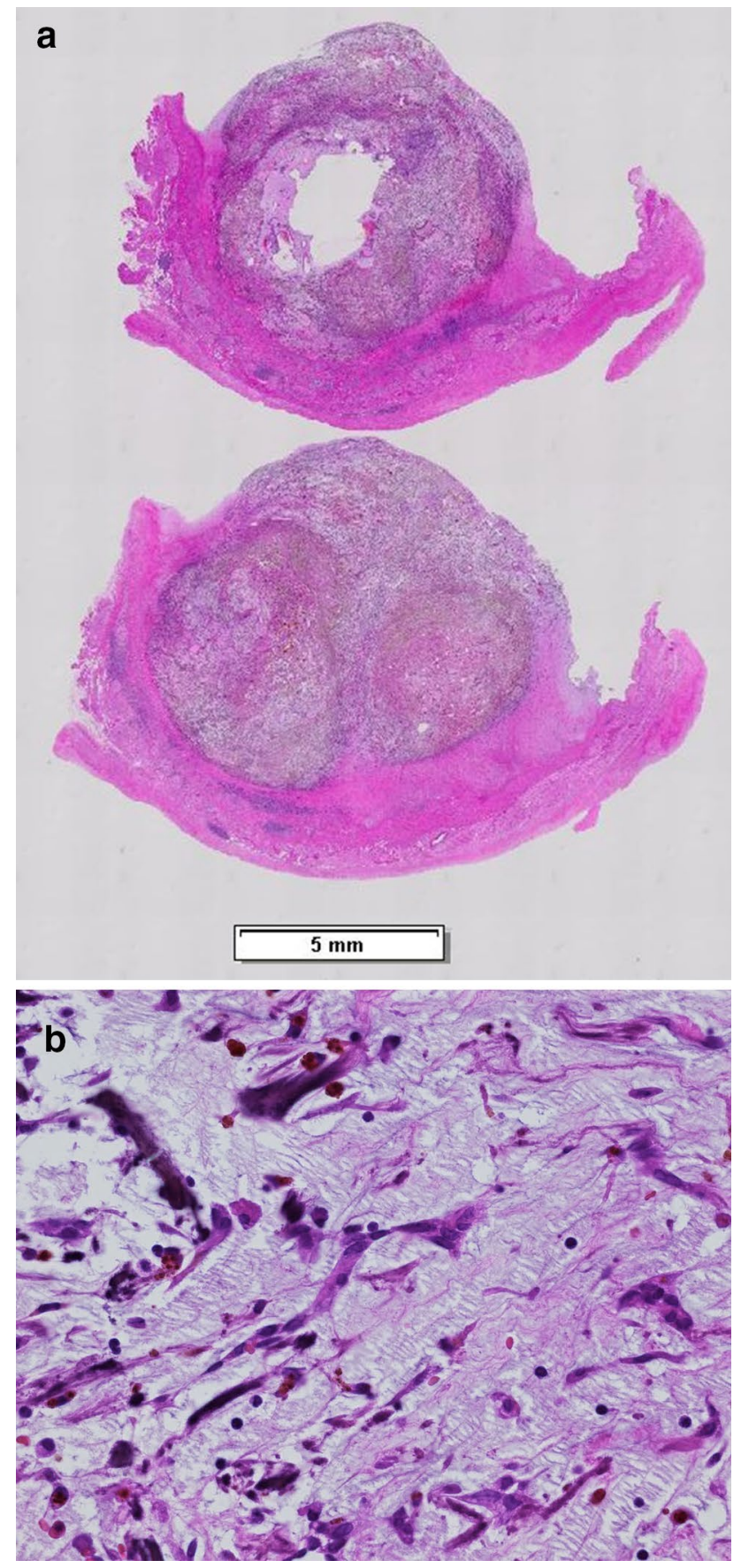

Fig. 3 a Loupe view of the resected tumor (hematoxylin-eosin stain). b Histopathological examination (hematoxylin-eosin stain) revealed small circular and spindle-shaped tumor cells in the mucous stroma, in addition to hemosiderin deposits and linear calcification (GamnaGandy bodies)

are benign, while $30 \%$ are malignant. Myxoma is the most common pathology among benign cardiac tumors, commonly occurring in the left atrium. Symptoms include shortness of breath, fainting, and dizziness, which arise due to embolism or hemodynamic disruption caused by mitral

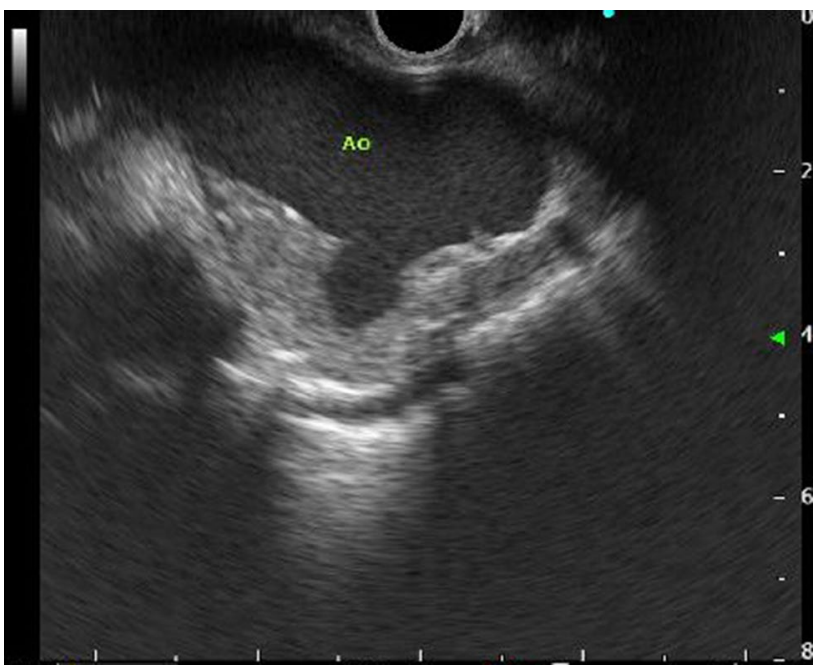

Fig. 4 Endoscopic ultrasound from the esophagus revealed aortic wall thickening and a sac-like protrusion of the aortic lumen. Ao aorta

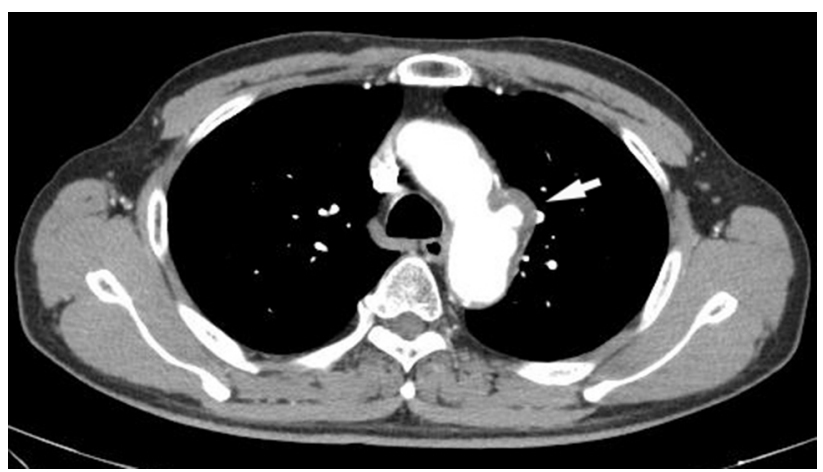

Fig. 5 Enhanced computed tomography demonstrated a saccular aneurysm in the aortic arch (arrow)

valve blockage. There is also a possibility of sudden death if a tumor is affixed to the mitral valve. Echocardiography is useful for diagnosing this condition, in which echogenic luminosity is uneven and sometimes accompanied by calcification. Generally, surgical resection is performed regardless of the tumor size. On the other hands, although aneurysm of the thoracic aorta can cause symptoms such as hoarseness, bloody sputum, and back pain, the majority of cases are asymptomatic. In many cases, the condition is diagnosed by simple chest X-rays or CT examinations for other diseases, with CT examinations being most useful for diagnostic purposes. Once a thoracic aortic aneurysm ruptures, the patient faces a high mortality rate, and surgical treatment must be considered based on the shape of the lesion, size of the aortic aneurysm, rate of expansion, observed symptoms, and other factors. Since saccular aneurysms and pseudoaneurysms are at a higher risk of rupture, surgery is indicated for these conditions. The present case involved a saccular aneurysm, and 
accordingly surgery was performed as this was the indicated treatment method.

During a literature review, there have been several reports [3-7] regarding incidental diagnosis of other diseases than the gastrointestinal one. For example, the report by Zalts et al. [3] involved a giant left atrial myxoma discovered unintentionally during examination of a gastric submucosal tumor. The small number of such reports regarding incidentally discovered cardiovascular diseases is probably because of few institutions where mediastinal observation during EUS examinations are actively performed. The number of EUS examinations performed is likely to increase in the future and the number of unintentionally discovered cases of cardiovascular disease during EUS examination is also likely to increase. Two cases out of 317 EUS examinations in our report suggest the importance of observational cardiovascular screening from the esophagus even in cases where EUS is performed to closely examine gastrointestinal disease. Screening of the cardiovascular and mediastinal lymph nodes from the esophagus requires only 1 min approximately. As cardiovascular diseases are often life-threatening, as in the present 2 cases, observational screening of the cardiovascular system from the esophagus should also be performed during EUS examinations just as the pharyngeal region is examined during upper gastrointestinal endoscopy.

\section{Compliance with ethical standards}

Conflict of interest The authors declare that they have no conflict of interest.

Ethical standards All procedures followed have been performed in accordance with the ethical standards laid down in the 1964 Declaration of Helsinki and its later amendments.
Informed consent Informed consent was obtained from all patients for being included in the study.

Open Access This article is distributed under the terms of the Creative Commons Attribution 4.0 International License (http://creativeco mmons.org/licenses/by/4.0/), which permits unrestricted use, distribution, and reproduction in any medium, provided you give appropriate credit to the original author(s) and the source, provide a link to the Creative Commons license, and indicate if changes were made.

\section{References}

1. Arima M, Tada M. Endoscopic ultrasound-guided fine needle aspiration biopsy in esophageal and mediastinal diseases-clinical indications and results. Dig Endosc. 2003;15:93-9.

2. Larghi A, Lococo F, Mainenti S, et al. EUS-guided fine needle tissue acquisition for the diagnosis of pleural metastases from endometrial cancer. Eur Rev Med Pharmacol Sci. 2014;18:1379-82.

3. Zalts R, Cohen O, Zohar Y, et al. Incidental diagnosis of cardiac myxoma by EUS. Gastrointest Endosc. 2015;82:1129-30.

4. Johal AS, Babich JP, Diehl DL. Left atrial thrombus incidentally discovered during EUS. Gastrointest Endosc. 2013;78:544.

5. Thosani N, Ferguson K, Buryanek J, et al. A rare case of incidental retroperitoneal seminoma diagnosed by endoscopic ultrasoundguided fine-needle aspiration. Endosc Ultrasound. 2014;3:66-7.

6. Vila JJ, Jimenez FJ, Irisarri R, et al. Prospective observational study of the incidental findings on endoscopic ultrasonography: should a complete exploration always be performed? Scand J Gastroenterol. 2009;44:1138-45.

7. Tee CT, Chen CC, Segarajasingam DS, et al. Pulmonary embolism diagnosed with EUS on a patient with adenocarcinoma of the pancreas (with video). Gastrointest Endosc. 2013;78:542-3. 